\title{
Software and hardware platform for testing of Automatic Generation Control algorithms
}

\author{
Alexey Vasiliev ${ }^{1}$, Artem Petrov ${ }^{1, *}$, and Sergey Svechkarev ${ }^{1}$ \\ ${ }^{1}$ National Research Tomsk Polytechnic University, 634050 Tomsk, Russia
}

\begin{abstract}
Development and implementation of new Automatic Generation Control (AGC) algorithms requires testing them on a model that adequately simulates primary energetic, information and control processes. In this article an implementation of a test platform based on HRTSim (Hybrid Real Time Simulator) and SCADA CK-2007 (which is widely used by the System Operator of Russia) is proposed. Testing of AGC algorithms on the test platform based on the same SCADA system that is used in operation allows to exclude errors associated with the transfer of AGC algorithms and settings from the test platform to a real power system. A power system including relay protection, automatic control systems and emergency control automatics can be accurately simulated on HRTSim. Besides the information commonly used by conventional AGC systems HRTSim is able to provide a resemblance of Phasor Measurement Unit (PMU) measurements (information about rotor angles, magnitudes and phase angles of currents and voltages etc.). The additional information significantly expands the number of possible AGC algorithms so the test platform is useful in modern AGC system developing. The obtained test results confirm that the proposed system is applicable for the tasks mentioned above.
\end{abstract}

\section{Introduction}

AGC systems must be constantly developed due to changes in market conditions, increasing degree of automation in the energy sector, development of computing systems, increasing number of Phasor Measurement Units (PMU), necessity of improving the reliability of power systems and changes in network configuration.

At the moment the AGC system of Russia operates on base of CK-2007 which is the main SCADA system used by the System Operator of the United Power System of Russia. Making changes to the settings and adding new algorithms requires testing the AGC system on adequate power system model. One of the complexes that allow real-time closed-loop testing of AGC system in composition with SCADA system is HRTSim, which was developed at the Tomsk Polytechnic University.

\footnotetext{
* Corresponding author: apsapetrov@gmail.com
} 


\subsection{HRTSim description}

The concept of HRTSim (Hybrid Real Time Simulator) simulation is based on the use of three modeling approaches: analog, digital and physical, each of which achieves maximum efficiency in solving individual subtasks. A detailed description of the concepts and its hardware application is presented in [1-3]. Modelling of primary processes in power equipment is done by analog computational units that solve differential equations describing this power equipment. Power system automatics are simulated on microcontrollers in hard real-time. Switching of equipment and interconnection of units into a general model of a power system is carried out on the physical level. Such a solution ensures continuous simulation of primary processes.

Adjusting of power system and power equipment parameters and automation settings as well as the continuous collection and processing of simulation results is carried out by specialized software - VmkServer.

VmkClient software allows to control operating conditions by changing the automatic setpoints values, switching devices positions and parameters of the generation equipment and the load. Also, it is possible to control the operating conditions according to the predetermined algorithms - the mode scenarios.

Interaction with third-party software or SCADA systems is done through the OffsiteClient component.

All the processes are simulated in real time, which allows to solve such problems as training of dispatch personnel, closed-loop testing of relay protection devices and local emergency control automatics, data exchange with SCADA systems for scientific, practical and educational purposes and much more.

\subsection{SCADA CK-2007 features}

Operational and informational software complex (OIC) CK-2007 is a high-performance real-time SCADA platform which is designed for receiving, processing, storing, and transmitting telemetric, reporting and scheduled information on the operation of energy facilities, networks and systems and provide flexible access to different users and external automated systems. The complex is widely used in Russian Federation as the basis for the construction of operational technological control and management systems in situational centers, data centers and control centers in the power industry for generating, network, marketing companies and system operators.

The complex is delivered with EMS, DMS and MMS applications in various configurations and also has Application Programming Interface (API) and Data Access Component (DAC) library which allows to implement additional modules to solve specific technological tasks [4-5].

\section{Implementation of AGC systems test platform}

The AGC test platform is composed of two complexes HRTSim and OIC CK-2007. The first plays a role of a controlled object - a power system, and provides an interface for reading/writing of operating parameters in real time via a specialized software interface and a user application. The second complex is the basis for building operational and technological management systems in the electric power industry, it collects state information from control objects, and provides all the necessary tools for implementing third-party modules for further processing. 


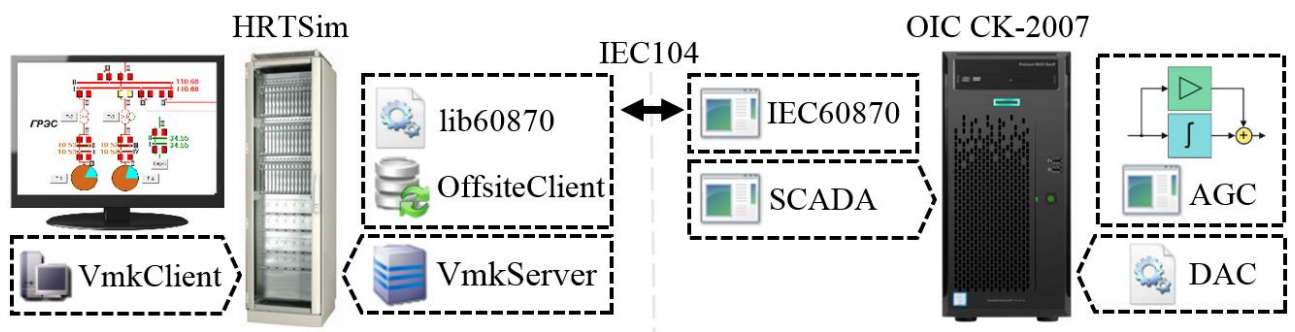

Fig. 1. Automatic Generation Control systems test platform

On the base of OffsiteClient two-way information exchange between HRTSim and OIC has been set up according to the IEC 60870-5-104 protocol, which makes it possible to transmit state information to OIC and to implement remote control of the generators that are modelled in the HRTSim.

Support for the IEC 870-5-104 protocol was implemented using the lib60870.NET library written in $\mathrm{C \#}$. The library is free to use for testing, evaluation, educational purposes and open-source projects under the terms of the GPLv3 license, for commercial projects commercial license is needed [6]. The library allows to implement both a client and a server which can exchange telemetry data, interrogation, time synchronization and control commands. Also a $\mathrm{C}$ version of the library is available.

The telemetry data is transmitted once a second or by interrogation command from a controlling station (OIC). The data is being filtered so the repeating values are not retransmitted. It is possible to configure dead band in order to reduce excessive traffic between HRTSim and OIC and to test the impact of accuracy of telemetry data coming to SCADA system on AGC system operation. Datasets for transmission and reception are configured using the graphical user interface shown in Figure 2.

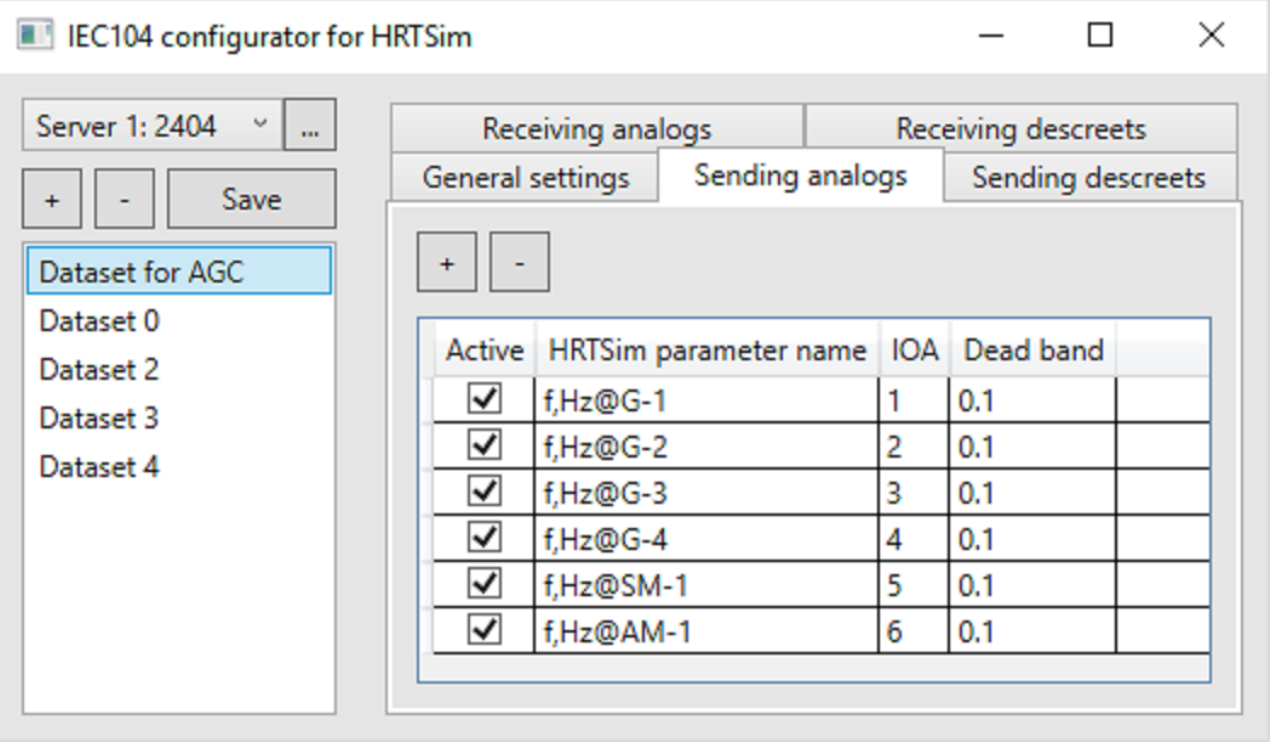

Fig 2. IEC 60870-5-104 protocol configurator for HRTSim

An AGC system testing process is carried out in two stages: preparatory and basic. At the first stage, the test object and testing facilities are being prepared:

- The power system model configuration;

- Development and verification of testing scenarios; 
- Preparation of the AGC system (transferring an existing AGC system to the test platform and adding of new parameters and algorithms);

- Configuration of two-way communication protocol;

- Setting up of OIC tools to record state variables and AGC system control actions.

At the second stage, all test scenarios are run sequentially, the results are analyzed, and a conclusion about the readiness and applicability of the AGC system are made.

\section{Results}

As was already mentioned above, in addition to practical tasks related to the interaction with the unified AGC system of Russia, research tasks can be solved. As an example of a research task, an external program for OIC CK-2007 has been developed that partially reproduces the functionality of the AGC system. The program implements the PI-regulator algorithm that calculates the change of active power generation that is needed to maintain the active balance at the frequency of $50 \mathrm{~Hz}$. The obtained value is distributed between the generators under AGC according to the given proportional coefficients and recorded in the CK-2007. When a variable in CK-2007 corresponding to the output of any of the generators updates, its value automatically sends via the IEC 60870-5-104 protocol and records in the HRTSim through OffsiteClient.

The AGC program is written in C\# using the .NET Framework 4.6.1. The reading and writing of information from the OIC is carried out using the Data Access Component library (DAC), which is included in CK-2007 complex. All necessary regulatory parameters involved in the process of operation of the AGC system are put on the form of the program, which allows you to customize the operation of the system for the needs of a particular task, and also use the developed program for scientific and educational purposes.
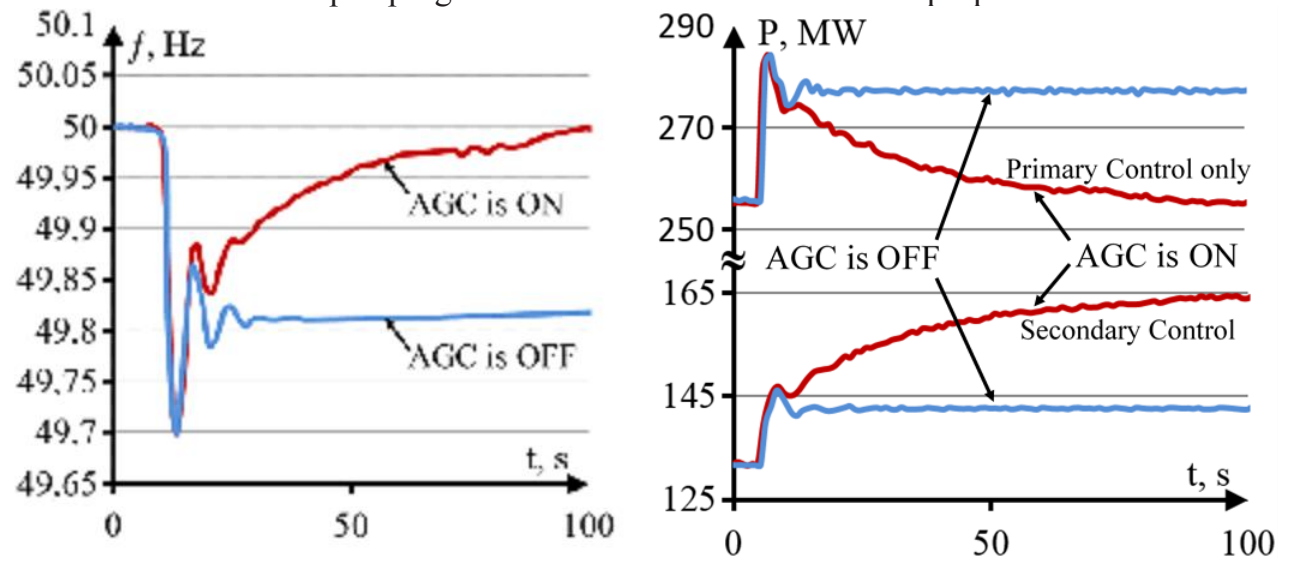

Fig. 3. Frequency and active power response on load switching of generators participating in the primary and secondary control

For example, in Figure 3 graphs of frequency and active power response on load switching of generators participating in the primary and secondary control are shown. The experiment was done twice - with AGC system turned on and off. As can be seen from the graphs, the developed program successfully restores the frequency of the power system that is modelled in the HRTSim to a value set by the setpoint, the processes occurring during the primary and secondary frequency control are fairly accurately reproduced. The value of the frequency exceeded the permissible limit of $50 \pm 0.2 \mathrm{~Hz}$ in the Russia, but recovered to an allowable value of $50 \pm 0.05 \mathrm{~Hz}$ in about 40 seconds. The results are explained by the small time constant of the AGC and the low capacity of the modelling power system (even a 
small change in the load power creates a significant relative imbalance), which does not allow to fully apply the requirements described in State Standard R 55890-2013 [7] to the power system being modeled in HRTSim.

\section{Conclusion}

The use of software and hardware tools mentioned above for modeling real-time processes in power systems allows testing control algorithms of AGC systems in a closed loop (including the AGC system that is used in operation in Russia). Testing of AGC systems together with the SCADA that is used in operation allows to simplify the transfer of AGC parameters from the test server to the real one and vice versa by automating the process and avoiding transfer errors.

Available in HRTSim extended set of primary state variables, in comparison to the real power system, allows to perform research tasks. CK-2007 Application Program Interface allows to implement third-party software applications not only of AGC systems, but also of other centralized automation systems, for example, Centralized Automatic Emergency Control or Centralized Voltage Control systems. Interaction via standard protocols and the availability to remotely launch VmkClient allows to territorially distribute the system and remove the function of technical support of HRTSim from the end user of the system.

\section{References}

1. A. Prokhorov, Yu. Borovikov, A. Gusev, Int. J. Smart Grid Clean Energy, 1 (1), 67 (2012)

2. N.Y. Ruban, A.O. Sulaymanov, R.A. Ufa, I.A. Razzhivin, MATEC Web Conf. 91, 1 (2016)

3. M.V. Andreev, Y.S. Borovikov, N.Y. Ruban, 11th Int. Forum Strategic Technol. 187 (2017)

4. JSC "Monitor Electric", official website. Available at: http://www.monitel.ru/ (accessed: 5.11.2017)

5. Operational and informational software complex CK-2007C, User manual, JSC "Monitor Electric", (2013)

6. IEC 60870-5-104 Libraries, MZ Automation, official website. Available at: http://mz-automation.de/index.php/products/iec-60870-5-104-protocol-libraries/ (accessed: 5.11.2017)

7. GOST R 55890 - 2013. Operativno-dispetcherskoe upravlenie. regulirovanie chastoty i peretokov aktivnoj moshhnosti. Normy i trebovaniya [State Standard R 55890 - 2013. Operational dispatch control. Load frequency control. Norms and requirements.], (2014) 